\title{
NOTES ON QUANTUM THEORY.
}

\author{
The Distribution of Thermal Energy.
}

By Gilbert N. Lewis and Elliot Q. Adams.

T $\mathrm{N}$ a previous paper ${ }^{1}$ we have made use of a hypothesis called "the theory I of ultimate rational units," to show that the interesting quantity, " $h$," which has come to be known as the "Wirkungsquantum," is, when expressed in suitable units, merely the square of the electron charge, multiplied by a simple numerical factor. This relation, in view of the important role of the constant " $h$ " in the development of the quantum theory, leads to the inquiry whether the facts on which that theory rests cannot be explained by less radical assumptions.

The discontinuity of the radiation field postulated in the more extreme forms of the quantum theory leads inevitably to the assumption of a similar discontinuity in the field of a static charge. Such a conclusion would mean that the classical equations for the electromagnetic field possess only statistical validity. It is extremely doubtful, moreover, whether any of the proposed forms of the quantum theory can avoid these consequences, when carried to a full logical development. A theory which requires that an electric oscillator radiate energy at one amplitude and not at a slightly lower amplitude can hardly be brought into harmony with the accepted theories of the continuous field. According to the view now advocated by Planck, an oscillator in thermal equilibrium with its surroundings continues to have a finite energy of oscillation, even at the absolute zero of temperature. This view is equally at variance with the accepted views of electromagnetics and with those of thermodynamics.

Now, if there were no other way of explaining the very important facts to which attention has been called by the quantum theory, it would be proper to make such assumptions and to modify the body of physical theory in so far as might be necessary to render it consistent with them. But we believe that no such necessity exists.

The phenomena which must be considered fall into three classes: first, the distribution of energy in the spectrum of a black body; second, the heat capacity of material systems, and third, the photoelectric and photochemical effects.

1 Phys. Rev., N. S., 3, 92 (I9r4). 
Several phenomena of the first two classes prove beyond question the failure of the classical theorem of equipartition, according to which the total kinetic energy of any system in thermal equilibrium is equal to a universal constant multiplied by the absolute temperature and by the number of independent modes of motion possible to the system. This number of modes of motion may be also expressed as the number of data which suffice to determine the instantaneous momentum of every part of the system.

The third class of phenomena bears a less direct connection with the principle of equipartition, and we shall discuss these phenomena in another place. The present paper will deal therefore only with those portions of the quantum theory which are immediately related to the problem of the partition of energy.

The Distribution of Energy in the Spectrum of the Black Body.

The so-called Rayleigh formula for the distribution with respect to frequency of the energy of a hohlraum, is the one which corresponds to the simple equipartition theory. It may be derived most simply from the equation relating the energy, $U_{r}$, of a one-dimensional electric oscillator to the energy of the same frequency, $u_{\nu}$, in a hohlraum in equilibrium with it, namely,

$$
U_{r}=\frac{c^{3} u_{v}}{8 \pi \nu^{2}}
$$

$u_{\nu}$ being the derivative of the energy density of the hohlraum with respect to frequency. This equation is derived from classical electromagnetic theory, and while its complete validity for high frequencies may be doubted, there seem to be good grounds for believing that it cannot be held responsible for the flagrant departure of the Rayleigh equation from observed fact.

If the energy of the oscillator at the temperature, $T$, is $U_{r}=k T$, then the Rayleigh equation follows at once

$$
u_{\nu}=\frac{8 \pi}{c^{3}} k \nu^{2} T \text {. }
$$

This equation can be put in the form

$$
u_{\nu} d \nu=\frac{8 \pi}{c^{3} h^{3}} k^{4} T^{4} x^{2} d x
$$

where $x=h \nu / k T$ and $h$ is a constant which does not appear directly in the Rayleigh formula, but which has been inserted for the sake of comparison with other radiation formulae. In this form, the Rayleigh 
formula obviously satisfies the Wien displacement law, which must be regarded as an inevitable consequence of thermodynamics. It is, however, evidently untrue, in that it requires that $u_{v}$ increase without limit with increasing values of $\nu$, and gives for the total energy of a hohlraum, at any finite temperature, an infinite value. It has been suggested by Jeans that the Rayleigh equation may after all be valid for complete thermal equilibrium, but that such equilibrium never obtains in practice on account of the slowness with which it is established, and that the conditions which actually prevail represent a sort of false equilibrium. In discussing certain phenomena relating to specific heat we shall show the inadequacy of such an explanation.

To represent the actual distribution in the spectrum, Wien proposed the formula

$$
u_{\nu} d \nu=\frac{8 \pi}{c^{3} h^{3}} k^{4} T^{4} x^{3} e^{-x} d x .
$$

This equation agrees qualitatively and to a large extent quantitatively with the existing data concerning radiation. In the region of low frequency and high temperature it gives, however, lower values of $u_{\nu}$ than have been obtained experimentally. For this reason Planck proposed the equation

$$
u_{\nu} d \nu=\frac{8 \pi}{c^{3} h^{3}} k^{4} T^{4} \frac{x^{3}}{e^{x}-\mathrm{I}} d x .
$$

This not only is in better agreement with observation, but has the distinct theoretical advantage that it becomes identical with Wien's for large values of $x$ and with Rayleigh's for small values of $x$. Indeed we have at present many reasons for believing that these two equations possess complete validity as limiting laws, true respectively for $x=\infty$ and $x=0$. Aside from this it is difficult to judge whether the Planck equation can be regarded as having a theoretical basis. The various derivations of this formula offered by Planck are founded upon different forms of quantum theory which we regard as unsound in principle. But the simplicity of the equation recommends it, and although the experiments of Paschen ${ }^{1}$ and of Rubens and Kurlbaum, ${ }^{2}$ were not in complete accord therewith, we must nevertheless conclude that if Planck's is not the true radiation formula it represents an extraordinarily close approximation to the truth.

It must be noted, however, than an infinite number of equations may be set up, which would agree as well with the experimental facts as the

1 Ann. d. Phys. (4), 4, 277 (I901).

2 Ann. d. Phys. (4), 4, 649 (I90I). 
Planck formula. Expressing the distribution of energy in accordance with the Wien displacement law as

$$
u_{\nu} d \nu=\frac{8 \pi}{c^{3} h^{3}} k^{4} T^{4} f(x) d x,
$$

$f(x)$ is $x^{2}$ in the Rayleigh formula, $x^{3} e^{-x}$ in the Wien, and $x^{3} /\left(e^{x}-1\right)$ in the Planck.

The total energy density $\int_{0}^{\infty} u_{\nu} d \nu$, is therefore dependent at a given temperature upon $\int_{0}^{\infty} f(x) d x$, which has the value $\infty$ in the Rayleigh, 6 in the Wien, and $\pi^{4} / 15=6.49+$ in the Planck formula.

Boissoudy ${ }^{1}$ has suggested $f(x)=x^{2} e^{-x}(\mathbf{I}+x)$ for which $\int_{0}^{\infty} f(x) d x=8$.

This equation is not in as good agreement with the facts as that of Planck, but as examples of different types of functions which would represent the available data within the limits of experimental error, we may mention:

$$
\begin{aligned}
& f(x)=x^{2} e^{-x}\left(\mathrm{I}+x^{3}\right)^{\frac{1}{3}}, \quad \int_{0}^{\infty} f(x) d x=6.16+; \\
& f(x)=x^{3} e^{-x} \operatorname{coth} x, \quad \int_{0}^{\infty} f(x) d x=\left(\pi^{4} / 8\right)-6=6 . \mathrm{I} 7+; \\
& f(x)=x^{3} e^{-x}+x^{2} e^{-2 x}, \quad \int_{0}^{\infty} f(x) d x=6.25 \\
& f(x)=x^{2} e^{-x}\left(\mathrm{I}+x^{2}\right)^{\frac{1}{2}}, \quad \int_{0}^{\infty} f(x) d x=6.42+.
\end{aligned}
$$

All of these equations become identical at the two limits with the equations of Rayleigh and of Wien, and it is evident that an indefinite number of such equations might be devised which would fit the true distribution curve with any desired degree of accuracy. Whether the Planck formula is the true one we must regard as an open question.

Whatever the true form of the distribution equation may be, it is unquestionably not the one obtained from the equipartition of energy, according to which the heat capacity not only of the hohlraum, but of any continuum, is infinite. In other words, according to that principle a material continuum is regarded as equivalent to a system composed of an infinite number of particles of infinitesimal mass, each capable of independent motion of the type which Boltzmann called "molekular ungeordnet." But such a system of particles cannot legitimately be

${ }^{1}$ Comptes Rendus, 156 , I364 (1913). 
regarded as a continuum. A (physical) continuum must be defined as a region in which all properties vary continuously from point to point, and in which the properties at any point may be predicted with a probability approaching complete certainty when the corresponding properties at other points taken nearer and nearer to the given point are ascertained. Any system which we are justified in calling a continuum is one in which the motions of neighboring elements cannot be regarded as completely independent or unordered.

Let us consider a hohlraum permeated by electric and magnetic fields. If these fields are produced according to equations of accepted electromagnetic theory the hohlraum is a continuum, and the magnitude of the electric and magnetic vectors at any point will differ in magnitude and direction from those at any neighboring point by an amount which becomes indefinitely less as the two points are taken nearer and nearer together. Without knowing quantitatively how near to a point the vectors must be found in order to predict their values at that point within a certain probable error, we may nevertheless assert that starting from a given point each of these vectors must maintain a component in the original direction for a finite distance in every direction. If then any hohlraum containing radiant energy should have within it a charged body, this body would be acted upon through finite intervals of time by forces of the same general direction and would acquire a finite kinetic energy. If the hohlraum is in thermal equilibrium the average kinetic energy of the charged body will be that which belongs to it at that temperature. In other words, since the charged body acquires thermal energy from the hohlraum the latter cannot have an infinite heat capacity.

We must assume, not that a continuum must have an infinite heat capacity, but that the very definition of a continuum precludes this possibility. We may state this principle in another way, in the case of the hohlraum. The electric and magnetic vectors at a point determine the general magnitude and direction of the vectors in neighboring points. The existence of radiant energy of infinite frequency is precluded, for in such radiation it would be impossible to determine the electromagnetic vectors at a point from those at any other point, however near together these points might be. The failure of the Rayleigh radiation formula may therefore be ascribed not to discontinuity of the hohlraum, as in the quantum theory, but to its very continuity.

The hohlraum thus interpreted as a continuum presents a very interesting problem which we may state, somewhat inadequately perhaps, as follows:

In any continuum containing vector fields the knowledge of the mag- 
nitude and direction of a vector at a single point determines a most probable value of the vector at any other point. In the simplest case this probable value would be a vector in the same direction, and with a magnitude diminishing with increasing distance from the given point, to zero at infinite distance. The probable value will in general be a function only of the magnitude of the original vector and of the distance.

In a given hohlraum therefore the electric or magnetic vector at each point determines a probable value of the vector at every other point, and if the average value of the magnitude of the vector were known throughout the whole region considered, and if the probability function which we have mentioned above were also known, the determination of the macroscopic condition of highest probability in the system would present no difficulties except those of an analytical character. In other words, a knowledge of this probability function ${ }^{1}$ is mathematically sufficient to give the true equation for the distribution of energy in the hohlraum.

We may call attention at this point to an interesting consequence of the theorem that the electric (or magnetic) vector in a hohlraum continues, for a finite distance from a given point, to have a component in the same direction as the vector at that point. Consider a hohlraum in thermal equilibrium at a given temperature, and some direction, say north, arbitrarily chosen. Choosing some point where the electric vector has a northerly component, the locus of the termini of lines drawn from this point and terminating at points where the north component disappears is a closed surface, and the whole volume of the hohlraum may thus be divided into a finite number of domains, in each of which either all the electric vectors possess a northerly component, or all possess a southerly component.

The average volume of these domains is obviously independent of the fixed direction chosen, and in fact depends on the temperature alone. It follows readily from the Wien displacement law that this average volume is inversely proportional to the cube of the absolute temperature. In other words, the average entropy of one of these domains is the same under all conditions of thermal equilibrium.

It is extremely interesting to note that from our theory, which may be considered in many respects the opposite of a quantum theory, we are thus led to a consideration of domains which are closely analogous to the "Elementargebiete" which Planck employs so frequently.

Another parenthetical observation may not be out of place here. A single molecule (at a given instant) cannot be regarded as a macroscopic

1 The possibility must be considered that this probability function may depend upon the average strength of the field, as it would, for example, in case the continuum were an elastic solid, subject to severe strains. 
system, as Planck uses this term. Only when enough molecules are taken to enable us to deal satisfactorily with averages can the system be so regarded. Now the interesting question arises: what is the minimum volume of a hohlraum in which the instantaneous conditions may be treated statistically without any great danger of error? The answer to this question is that a hohlraum, in order to be regarded as a macroscopic system at any instant must contain a considerable number of these elementary domains. ${ }^{1}$

Returning now to the consideration of the probability function, discussed above, we may, if it is desirable, consider the energy of the hohlraum resolved by a Fourier analysis with respect to frequencies. This convenient, although by no means necessary, resolution enables us to divide the radiation into a number of approximately monochromatic constituents. Now it is evident that the probability function due to the character of the hohlraum as a continuum is without effect upon the very long wave-lengths, but that it makes the existence of any given amount of energy at smaller and smaller wave-lengths less and less probable. This new factor of probability, which could be derived from the other probability function, if that were known, by mere mathematical transformation, may be regarded as the factor which multiplied by the $u_{\nu}$ of the Rayleigh formula gives the true radiation formula. Thus, if Planck's formula is correct, this factor is $x /\left(e^{x}-\mathrm{I}\right)$, where $x$ is, as before, $h \nu / k T$. In any case this factor, according to the Wien displacement law, must be a function of $\nu / T$ only.

Since free space maintains undamped any electromagnetic disturbances which may be imposed upon it, it may seem strange to regard the properties of a hohlraum in thermal equilibrium as in any way intrinsic, but that it may be so regarded is shown by the fact that the properties of a hohlraum are entirely independent of the nature of the walls or of the mechanism of radiation. Nevertheless we may hope to obtain important information concerning the hohlraum from the laws of the distribution of energy in material systems.

The Distribution of Thermal Energy in Material Systems.

There are two important cases in which the equipartition law gives a quantitative explanation of observed specific heats. The heat capacity

\footnotetext{
1 The volume of one of these elementary domains can be roughly estimated by assuming that in ultimate rational units the average volume of the elementary domain is unity at unit temperature. Except for the factor $4 / 3$, this is equivalent to assuming that the average entropy of any elementary domain is unity (or $k$ in ordinary units). This would make the volume of the elementary domain of the ordcr of $10^{-2}$ c.c. at one centigrade degree above the absolute zero. A value of the same order of magnitude is obtained by assuming that the volume of the elementary domain at any temperature is the cube of $\lambda_{m}$, that is, the wave. length of maximum energy at that temperature.
} 
of a monatomic gas is precisely that which is calculated from the assumption that the atoms possess no energy except kinetic energy of translation; and the heat capacity of most solid elements at ordinary temperatures is that calculated from the assumption that each atom possesses this same translational energy and, on the average, an equal amount of potential energy. There is, however, nothing apparent in the derivation of the equipartition law which would lead us to expect the heat capacity of solids to diminish at lower temperatures, approaching zero at the absolute zero of temperature, nor which would account for the failure of the atom to acquire energy of rotation as well as energy of translation.

Such departures from the principle of equipartition Jeans formerly attempted to interpret as due to the non-attainment of true thermal equilibrium. According to this theory a monatomic gas at a given temperature would rapidly attain the appropriate translational energy, but would acquire rotational energy, and other forms of energy within the atom, only with extreme slowness. The following argument seems to us sufficient to show the inadequacy of this explanation. A monatomic gas, like argon, has a dielectric constant higher than unity. This means that in an electric field certain modes of motion, of rotation or displacement, manifest themselves in the atoms. An atom of argon therefore, subjected to the electromagnetic field of radiation at a given temperature, must respond rapidly in these modes of motion, which apparently, however, make no appreciable contribution to the heat capacity. Moreover, that theory could in no possible way account for the diminution in the heat capacity of solids at low temperatures, for it would have to be admitted in any case that in the region of validity of Dulong and Petit's law the establishment of true temperature equilibrium is rapid. Now as the temperature is lowered the theory would require that at some temperature the establishment of thermal equilibrium would take place with a very small but measurable speed; such a phenomenon, if existent, would certainly have been observed.

The situation seems to be this: a particle subject to no constraints acquires at each temperature the kinetic energy demanded by the equipartition theorem, but a constraint in any mode of motion diminishes the kinetic energy associated with that mode of motion and this diminution is greater the more powerful the constraint. ${ }^{1}$ This is in brief the phenomenon for the explanation of which the quantum theory has been invoked. Before proposing a different explanation we may put the problem in another form.

It is customary to correlate with the energy of a hohlraum the energy

${ }^{1}$ We use the word constraint in its most general sense, to mean any set of forces which operate against the free acquisition of rectilinear motion. 
of a system of electrical oscillators by means of equation ( $\mathrm{I}$ ), connecting the energy of an oscillator of frequency, $\nu$, with the energy density of a hohlraum associated with the same frequency, and this is the method by which Einstein was led to the first formula for the heat capacity of solids at low temperature. The approximate validity of this and other similar formulæ has been regarded as a substantiation of the quantum theory, but the authors of these formulæ have recognized that their derivation depended only upon the assumption that the Planck formula is a satisfactory empirical equation for the distribution of energy in the hohlraum,

By combining equation ( $\mathrm{I}$ ) and the Wien displacement law, equation (6), we obtain for the energy of any electrical oscillator

$$
U_{r}=k T F(x),
$$

where $F(x)$, as we know from a study of the spectrum, approaches unity for small values of $x$, that is, at high temperatures or low frequencies, but approaches zero at low temperatures and high frequencies. It has been universally assumed, and we think with perfect propriety, that there is nothing unique about an oscillator which happens to be charged, and that this same equation would hold for any mechanical oscillator. Hence, any oscillator of given frequency will have at high temperatures the energy predicted by the equipartition principle, but zero energy at the absolute zero, where $F(x)=0$.

Now while it is permissible to regard a material system as composed of a large number of simple harmonic oscillators of definite frequencies, this resolution is not necessary nor is it perhaps always advantageous. Thus Debye employs this method of representing the conditions prevailing in a solid body as a mere mathematical expedient, but regards his system as a quasi-continuum, pervaded by chaotic thermal vibrations.

In general a process which is associated with a definite frequency cannot be regarded as elementary, for in periodic motion a system may pass through very different conditions of velocity and acceleration in a single period. If a system be divided into elementary parts, each undergoing motions which are in general periodic it is entirely justifiable to make a Fourier analysis and consider the distribution of energy among frequencies, but it is perhaps more fundamental to arrange the elementary parts according to the instantaneous accelerations, and to determine the distribution of energy among the accelerations.

Such a resolution may be made in a number of ways but in general it will be true that just as the energy belonging to any frequency is less than that required by the equipartition law, and by a factor which is a function of $\nu / T$ only, so also the energy of any portion of the system is on 
the average less than equipartition demands and by an amount which is greater, the greater the acceleration of that portion of the system, and the lower the temperature.

In so far as this conclusion is based upon the Wien displacement law it may be regarded as thermodynamic in character and independent of the specific constitution and properties of any particular system, but at most thermodynamics can only tell us that the factor in question must be of this form if it exist. What change, therefore, must be made in the derivation of the partition law to permit the entrance of this factor involving acceleration?

We believe that the true answer to this question is suggested by our previous remarks concerning the continuum. The deviations from the equipartition law occur in systems subject to powerful constraints, and in such a system the motion of its parts can not be regarded as independent. In a system of mathematical particles such an assumption might be justifiable, but in a system of actual particles each particle determines a field of force and to these fields must be attributed some part of the mass of the system. We are dealing therefore not with a mere assemblage of discrete particles nor with a pure continuum, but with a quasi-continuum which approximates the one or the other according as the character of the constraints and the nature of the problem emphasize the singular points which are the atomic centers or the field in which these centers are, as it were, imbedded.

It has frequently been assumed that the failure of the equipartition law will necessitate at the very least some striking amendment of the accepted principles of mechanics. It seems to us, on the contrary, that the fault is not in these principles but in their application.

In the derivation of the equipartition theorem it is assumed that the idea of a collision is unambiguous. In the ideal collision identifiable objects of definite mass ${ }^{1}$ exchange momenta according to simple laws, but any actual collision presents a far more difficult problem. The particle concerning which we possess the most specific information is the electron, and yet it has hitherto been impossible to solve the simple problem of the motion of two electrons which at a given distance are started toward one another in a straight line with a known velocity. In certain limiting cases we are justified in regarding the mass of the system as localized at the electron centers, but in general it must be regarded as distributed throughout the infinite electron fields. Likewise the localization of the mass of any particle at the center of the particle leads in some

${ }^{1}$ We are in no way concerned here with the change in mass with velocity demanded by the principle of relativity. The deviations from the principle of equipartition are especially pronounced at the lowest temperatures where the smallest velocities are concerned. 
cases to no confusion, but this is not the case when the fields of force of the particles appreciably overlap. In the case of a quasi-continuum, where mutual constraints are in evidence, some part of the mass of the system must be assigned to the fields of these constraints.

The fact that these considerations have been ignored is, in our opinion, alone responsible for the erroneous theorem concerning the partition of energy, and while we are still unable to offer any quantitative solution of this difficult problem we believe that all the phenomena of the distribution of thermal energy may be qualitatively and semi-quantitatively explained if we assume that, in a system subject to constraints, the acceleration of any portion of the system under given conditions is less than it would be in the absence of the constraints, and that this difference increases with the acceleration. This does not mean that we must give up the law that acceleration is equal to force divided by mass, but only that in a quasi-continuum it is hard to localize the forces and masses, and that formerly we have assumed a given mass to be acted upon by a larger force than the actual one.

Without attempting to analyze the situation further let us state once more our proposition, namely, that every portion of a system in thermal equilibrium possesses on the average a kinetic energy which is less than that given by the equipartition law by an amount which is larger, the greater the constraints, the higher the instantaneous acceleration, and the lower the temperature. We may now proceed to apply this general principle to specific cases.

The Heat Capacity of Solids.-According to the principle just enunciated, the deviation from the law of Dulong and Petit should be greater the lower the temperature, and at a given temperature should depend upon two factors, namely, the rigidity of the constraints acting upon the atoms and the mass of the atom. For the smaller the mass of the atom, the greater its average acceleration must be, in movements of a vibratory character, to give it a certain amount of energy.

In fact all of the elements which depart appreciably from the law of Dulong and Petit at ordinary temperatures are those of small atomic weight, and the element which shows the greatest deviation of all is carbon in the form of diamond, in which the internal constraints must be enormously greater than in the case of any other element. Boron is another element of high internal rigidity, and its heat capacity is about the same as that calculated for hydrogen in the solid state (at room temperature). What hydrogen lacks in rigidity it makes up in the smallness of its atomic weight.

The element which above all others is characterized by small atomic 
mass is electricity, and we believe that the failure of the electrons to contribute materially to the heat capacity of a metal is merely a deviation from the law of Dulong and Petit which differs in magnitude but not in kind from those which are already familiar. We are thus able to understand why the electrons in metals which from some points of view appear to be free, still contribute nothing to the heat capacity. Their mass is so extremely small that very weak constraints prevent their acquiring an appreciable amount of thermal energy at ordinary temperatures, at high temperatures we must expect them to increase the heat capacity of the metals.

The Heat Capacity of a Monatomic Gas.-Assuming that the moment of inertia of an atom is extremely small, any appreciable amount of rotational energy must be associated with very great acceleration, and therefore at any ordinary temperature the energy of rotation will be no considerable part of the thermal energy of a monatomic gas. In fact, Pier $^{1}$ has shown that the heat capacity of argon is constant within the limits of experimental error up to $2,350^{\circ} \mathrm{C}$. If the moment of inertia of the atom were known, it would be possible to estimate the temperature range in which the heat capacity of a monatomic gas would pass from the value at lower temperatures to double that value. It is probable however that before the atoms acquire much rotational energy they will acquire an inner energy due to the vibration of their parts. Certain metallic vapors radiate thermally below $\mathrm{I}, 000^{\circ} \mathrm{C}$. While the internal oscillations necessary to produce radiation may be associated with comparatively little energy, we should expect the heat capacity of a monatomic gas to increase soon after it begins to produce appreciable thermal radiation.

The Heat Capacity of a Diatomic Gas.-According to the principle of equipartition the molal heat capacity at constant volume of a diatomic gas should be ${ }_{3}^{3} R$ if the molecule can possess only translational energy, ${ }_{2} / 2 R$ if it may also rotate about the two axes perpendicular to the line of centers of the atoms, and $7 / 2 R$ if it further possess kinetic and potential energy of vibration in the line of centers. According to the experiments of Eucken ${ }^{2}$ the heat capacity of hydrogen gas is approximately ${ }^{3} / 2 R$ at all temperatures below $50^{\circ}$ absolute; above that temperature it rises rapidly and then more slowly, approaching at about room temperature the value ${ }^{5} / 2 R$. From this point on the heat capacity increases linearly with the temperature up to the highest temperature where it has been investigated, $2,000^{\circ} \mathrm{C}$. It has ordinarily been assumed, we believe correctly, that the heat capacity at ordinary temperatures is

1 Pier, Z. Elektrochem., 15, 536 (r909).

${ }^{2}$ Eucken, Ber. Berl. Akad., Igr 2, I4I. 
due to translational and rotational energy and not, to any large extent, to vibrational energy. The rotational energy apparently obeys the equipartition theorem above room temperature, but falls rapidly to zero with diminishing temperature. The attempts which have been made to explain this phenomenon by means of the quantum theory have been extremely unconvincing. The association of a certain amount of energy with a certain frequency has been an essential part of the quantum theory, but a rotating molecule possesses no natural period, and we could hardly expect the application of the quantum theory in this case to prove successful.

The principle which we have stated above affords a simple explanation of these phenomena. As in the case of the monatomic gas, we should expect the moment of inertia of the rotating molecule to determine the degree of departure from the equipartition law. If the various diatomic gas molecules have approximately the same dimensions, then a given energy of rotation of the hydrogen molecule corresponds to a higher acceleration than in the case of other gases, and we should expect hydrogen to be the first gas to approach in heat capacity ${ }^{3} /{ }_{2} R$ as the temperature is lowered. This appears to be the case.

Before the diatomic gases have attained their full energy of rotation, they apparently begin to have an appreciable energy of vibration, and the part of the heat capacity due to this cause appears to be a linear function of the temperature. Now in other cases which have been considered, the attainment of a new type of thermal energy with increasing temperature has initially a rate which increases rapidly with the temperature; thus Debye states the law that the heat capacity of a monatomic solid is proportional to the cube of the absolute temperature at low temperatures. We believe that this law is independent of the more specific assumptions from which he derived it, and that for all substances, crystalline or non-crystalline, when the inner constraints are large and the temperature low, the heat capacity is proportional to the cube of the temperature. In other words, this is the general property of a continuum, which quasi-continua approach as a limit. To this conclusion that the heat capacity of any continuum would follow the same law as that of the hohlraum we were first led by considerations analogous to those in our paper on the ultimate rational units. A very interesting derivation of this law has just been obtained by Professor Tolman from his principle of similitude. ${ }^{1}$

The heat capacity of a three-dimensional continuum is proportional to the cube of the temperature; that of an $n$-dimensional to the $n$th power

${ }^{1}$ Phys. Rev., N. S., 4, I45, I9I4. 
of the temperature. A diatomic molecule capable of vibration only in the line of atomic centers approaches with increasing strength of constraint and decreasing temperature an ideal one-dimensional continuum. The part of the heat capacity of a diatomic gas which is due to vibration should therefore be a linear function of the temperature over a considerable range. ${ }^{1}$

The departure from the value predicted from equipartition of the heat capacity due to vibration should be the least in those cases where the largest amount of energy can be reached with the smallest average acceleration, that is, where the constraints are least and the masses greatest. Now iodine has not only the highest atomic weight of all the diatomic gases which have been investigated, but also the lowest internal constraints, as shown by the fact that it dissociates at a lower temperature than any other diatomic gas. In fact, iodine has the highest heat capacity of all the diatomic gases.

Chemical Laboratory of the University of California, Berkeley, May 22, I9I4.

${ }^{1}$ Le Chatelier found that the molal heat capacity of diatomic gases at constant volume could be adequately represented over a wide range of temperature by the equation $c_{v}=$ $4.5+a T$, and later measurements have been in pretty good agreement with this formula. According to the theory stated above the first constant of this formula should be $5 R / 2$ or nearly 5.o. The difference is probably due to the fact that owing to the scarcity of experimental data at higher temperatures the values at room temperatures have been given great weight and some of the diatomic gases probably do not acquire their full rotational energy until somewhat higher temperatures are reached. 